\title{
About BPAS
}

The British Pregnancy Advisory Service (BPAS) supports reproductive choice by advocating and providing high-quality, affordable services to prevent or end unwanted pregnancy with contraception or by abortion.

\section{Our values}

MISSION STATEMENT

The British Pregnancy Advisory Service was established as a registered charity in 1968 to provide a safe legal abortion service. At that time, shortly after the legalisation of abortion, the National Health Service (NHS) was unable to provide abortion care for all who needed it. Today NHS abortion services remain limited and so BPAS continues to provide affordable, high-quality care for those who cannot, or choose not, to access the NHS. The British Pregnancy Advisory Service collaborates closely with the NHS and, in areas where local arrangements allow it, BPAS provides a range of reproductive health care services, free of cost to the user, on behalf of the NHS. The British Pregnancy Advisory Service is now Britain's largest single abortion provider and cares for almost 50,000 women with unwanted pregnancies each year.

The British Pregnancy Advisory Service has a commitment to education, research and the development of good practice. It is a source of accurate information and informed comment about the causes, consequences and management of unwanted pregnancy to parliamentarians, policy makers and opinion formers.

BPAS BELIEVES THAT

- currently available methods of contraception cannot prevent all unintended pregnancies and legal abortion is necessary if women are to regulate their fertility

- contraception and legal abortion are an essential part of health care and should be freely available to all through a publicly funded NHS

- reproductive health services offered by specialist agencies such as BPAS are an essential supplement to the NHS if all women with unwanted pregnancies are to have early access to affordable specialist care.

BPAS EXISTS TO

- provide support and care for women seeking legal abortion

- inform and educate policy makers, opinion formers, the media and the public on issues relating to unwanted pregnancy

- deliver contraceptive advice and care including male and female sterilisation and emergency contraception in collaboration with existing NHS services
- promote and encourage research, and the advancement of science and understanding, in matters concerning the regulation of fertility

- advocate the need for safe legal abortion.

BPAS AIMS TO

- provide reproductive health care services that are responsive to the needs of those who might wish to use them

- promote the development of services that are accessible, effective, safe and confidential

- safeguard individual freedom and moral autonomy in making reproductive choices

- utilise its experience of abortion provision to contribute to the collective knowledge of those who provide reproductive health care.

\section{Governance}

The British Pregnancy Advisory Service is governed by a board of trustees who meet quarterly to determine policy and ensure that the organisation remains true to its charitable aims and values, and that charges to women or to the NHS are no greater than is necessary to cover costs and service development.

\section{Our services}

The British Pregnancy Advisory Service treats all clients with dignity and respect and provides confidential, non-judgmental services

ABORTION CARE

The British Pregnancy Advisory Service provides abortion care at eight clinics and four day-care units. Pregnancy testing, counselling and consultation to establish appropriate and acceptable care is offered at 33 consultation centres.

The British Pregnancy Advisory Service offers a choice of abortion methods appropriate to the gestation of the pregnancy. In early pregnancy, BPAS offers early medical abortion and vacuum aspiration procedures under local or general anaesthetic. In later pregnancies, clinics offer a surgical alternative to medical induction whenever possible.

The British Pregnancy Advisory Service provides abortion care on behalf of more than 60 NHS health authorities and trusts and is responsible for almost two-thirds of NHS-funded abortions undertaken by specialist agencies.

EMERGENCY CONTRACEPTION

The British Pregnancy Advisory Service was the first service provider to encourage its doctors to provide emergency "after sex" contraceptive pills (ECPs) in advance of a woman's need to use them. Doctors at BPAS consultation centres will also prescribe ECPs to women who have already risked 
pregnancy. A supply of ECPs is offered routinely to women attending BPAS clinics for abortion.

sterilisation and vasectomy

The British Pregnancy Advisory Service is able to provide female sterilisation procedures using local or general anaesthetic with the choice of day-care treatment or an overnight stay. Vasectomy is usually provided as a day-care procedure using local anaesthetic. Vasectomy reversal also available. Both procedures are available regardless of age, parity or marital status.

specialist family planning services

In some areas BPAS provides a specialist contraceptive service by arrangement with the local NHS.

information

The British Pregnancy Advisory Service provides accurate, balanced information on matters relating to unwanted pregnancy. Briefing papers on relevant medical, legal, demographic and ethical issues are circulated to policy makers and opinion formers. The press office provides comment and support for journalists and researchers.

education

The British Pregnancy Advisory Service organises seminars and symposia for health professionals, administrators and policy makers to facilitate the dissemination of new ideas and research.
The British Pregnancy Advisory Service provides speakers for conferences and workshops and produces materials to assist students.

\section{POLICY FORMATION}

The British Pregnancy Advisory Service works closely with the individuals and organisations responsible for reproductive health care policies. These include the Royal College of Obstetricians and Gynaecologists and the Department of Health. The British Pregnancy Advisory Service uses its experience of caring for women with unwanted pregnancy to inform those responsible for public policy on reproductive health issues.

ADVOCACY

The British Pregnancy Advisory Service seeks opportunities to advocate the need for safe, legal abortion care for all those who wish to use it. The British Pregnancy Advisory Service supports those seeking to make abortion more easily accessible throughout Britain and those working for the legalisation of abortion in the North and South of Ireland. The British Pregnancy Advisory Service advises the all-party pro-choice group of parliamentarians and is a member of the Pro-Choice Alliance network. 